\title{
Adaptive Control of a Class of Switched Nonlinear System with Partial State Constraints Using a Barrier Lyapunov Function
}

\author{
Enchang Cui, Yuanwei Jing, and Xiaoting Gao \\ College of Information Science and Engineering, Northeastern University, Shenyang, China \\ Correspondence should be addressed to Enchang Cui; andcharm_cui@hotmail.com
}

Received 27 March 2017; Revised 29 June 2017; Accepted 16 July 2017; Published 28 August 2017

Academic Editor: Asier Ibeas

Copyright (C) 2017 Enchang Cui et al. This is an open access article distributed under the Creative Commons Attribution License, which permits unrestricted use, distribution, and reproduction in any medium, provided the original work is properly cited.

\begin{abstract}
This paper discusses partial state constraint adaptive tracking control problem of switched nonlinear systems with uncertain parameters. In order to ensure boundedness of the outputs and prevent the states from violating the constraints, a barrier Lyapunov function (BLF) is employed. Based on backstepping method, an adaptive controller for the switched system is designed. Furthermore, the state-constrained asymptotic tracking under arbitrary switching is performed. The closed-loop signals keep bounded when the initial states and control parameters are given. Finally, examples and simulation results are reported to illustrate the effectiveness of the proposed controller.
\end{abstract}

\section{Introduction}

There is a strong industrial background of switched system in various fields. And for exactly that reason, many researchers have discussed the theoretical and applied research of switched system, and some of them have achieved commendable results in the last decade [1-3]. Since nonlinearity is the nature of the universe, more and more attention of switched nonlinear system has been drawn by control field [4-6]. It is also worth mentioning that a class of switched nonlinear system in lower triangular form is considered in [7], of which the backstepping method is used and a common virtual control is constructed to achieve the aim. Nevertheless, relatively accurate parameters are required by most control strategy for switched nonlinear system. Apparently, these desirable demands could not be met in practical terms.

Constraints are important issues in the study of physical systems, the authors in $[8,9]$ emphasized this problem in nonlinear saturation and performance and safety specifications, respectively. Taking a practical example for illustrative purposes, the attitude-control mass expulsion system of electrostatic microactuators is confronted with constraints, owing to the necessity of both the position and the speed of the movable electrode to be controlled. However, the development of constrained control is restrained by meeting the practical requirement. In this scenario, the extensive attention of the design and the analysis of constrained control has been increased. Recently, barrier Lyapunov functions (BLFs) have been proposed to solve the control problem of nonlinear systems. The state of the system will be not contradictory to the constraints [10-13] utilizing BLFs. Although the BLF is proven to settle constrained control problems [14], the parameters of switched nonlinear systems are expected to be deterministic.

Moreover, parameter uncertainties are widespread in realistic systems; hereon it has already been reported that adaptive control is an effective method to deal with such uncertain. On the one hand, remarkable achievements have been obtained from the research on adaptive control of nonlinear system. In [15-17], for instance, the control method is proposed and the preferable control performances are provided aiming at nonlinear time-delay systems. On the other hand, many researchers study the adaptive control problem of switched systems with uncertain parameters [1820 ] with the development of adaptive control, and preliminary results have been obtained [21, 22]. In [23], an adaptive tracking controller is designed for switched stochastic nonlinear systems with unknown actuator dead-zone; the satisfying control performance is obtained as well. Among the aforementioned works, few authors have addressed the 
important issue that considering the uncertainty and the constraint together. Hence, the approach to design constraint adaptive controllers for switched nonlinear systems has been not reported to the best of our knowledge.

In the present paper, the adaptive tracking control problem of a class of switched nonlinear system with partial state constraints is solved. The progressive state tracking would not violate constraint conditions and all signals would be bounded when the parameter increases to infinitely great and approaches a certain value based on BLF. The control effectiveness of BLFs is verified by the comparative simulation results with quadratic Lyapunov functions (QLFs).

The remainder of this paper is organized as follows. Section 2 formulates the control problem. The partial state constraint problems of barrier Lyapunov functions are elucidated. Section 3 develops the adaptive controllers with state constraints; the stability analysis and the proof process are presented as well. Section 4 corroborates the expected effectiveness of the proposed controller by means of selected simulation results. Finally, Section 5 concludes this paper.

\section{Problem Statements and Preliminaries}

2.1. Problem Formulation. Consider a class of the switched nonlinear systems with uncertain parameters in the following form:

$$
\begin{aligned}
& \dot{x}_{i}=F_{i}^{\sigma(t)}\left(x_{i}\right)+\theta_{b}^{\sigma(t)} g_{i}\left(x_{i}\right) x_{i+1} \\
& \dot{x}_{n}=F_{n}^{\sigma(t)}\left(x_{n}\right)+\theta_{b}^{\sigma(t)} g_{n}\left(x_{n}\right) u \\
& y=x_{1},
\end{aligned}
$$

where $\theta_{b}^{i} \in R^{n}$ are the unknown piecewise constant parameters. $\sigma(t) \in P=\left\{1,2, \ldots, p_{s}\right\}$ is a non-Zeno switching signal which is right continuous. $u \in R$ is the control input. $F_{k}^{i}$ and $g_{k}$ are smooth vector fields with $F_{k}^{i}(0)=0$ and $g_{k}(x) \neq$ $0, \forall x \in R^{n}$.

In addition, $F_{i}^{j}\left(x_{i}\right), \forall j \in P$ is unknown switched nonlinear function which can be linearly parameterized as

$$
F_{i}^{\sigma(t)}\left(x_{i}\right)=\sum_{k=1}^{p} f_{k i}\left(x_{i}\right) \theta_{f_{k i}}^{\sigma(t)},
$$

where $f_{k i}\left(x_{i}\right)$ is smooth function and $\theta_{f}^{i} \in R^{n}$ is a vector of uncertain parameters satisfying $\theta \in \Omega_{\theta}$ with known compact set $\Omega_{\theta}$. The parameters $\theta_{b}^{i}$ and $\theta_{f}^{i}, \forall i \in P$, the switching time instants $T_{k}, k=1,2, \ldots$, and the switching index $\sigma(t) \in P=$ $\left\{1,2, \ldots, p_{s}\right\}$ are all unknown. There exist positive constants $P_{i}$ such that $\left\|f_{k i}\left(x_{i}\right)\right\| \leq P_{i}$ for all $\left|x_{i}\right| \leq b, i=1,2, \ldots, n$ due to smoothness property.

According to (1) and (2), system (1) can be transformed in to the following form:

$$
\begin{aligned}
\dot{x}_{i}=F_{i}^{\sigma(t)}\left(x_{i}\right)+\theta_{b}^{\sigma(t)} g_{i}\left(x_{i}\right) x_{i+1} & \\
=\sum_{k=1}^{p} f_{k i}\left(x_{i}\right) \theta_{f_{k i}}^{\sigma(t)}+\theta_{b}^{\sigma(t)} g_{i}\left(x_{i}\right) x_{i+1}, & \\
& i=\{1,2, \ldots, n\} .
\end{aligned}
$$

The control target is to design an adaptive controller for system (1) such that $y$ tracks a desired trajectory $y_{d}$ asymptotically; that is, $\lim _{t \rightarrow \infty}\left(y-y_{d}\right)=\lim _{t \rightarrow \infty} z_{1}=0$ and $\left|x_{i}\right| \leq b, b$ is a positive constant. Moreover, in order to make the problem here more tractable, we give the following assumption, which is common but practical.

Assumption 1 (see [24]). $\theta_{b}^{i}$ for all $i$ 's have the same sign and whose common lower bound is known, that is, $0<\bar{\theta}_{b}<\left|\theta_{b}^{i}\right|$.

\subsection{Preliminaries}

Definition 2 (see [12]). A barrier Lyapunov function (BLF) is a scalar function $V(x)$, defined with respect to the system $\dot{x}=f(x)$ on an open region $D$ containing the origin, that is, continuous, positive definite, has continuous firstorder partial derivatives at every point of $D$, has the property $V(x) \rightarrow \infty$ as $x$ approaches the boundary of $D$, and satisfies $V(x(t)) \leq b \forall t \geq 0$ along the solution of $\dot{x}=f(x)$ for $x(0) \in D$ and some positive constant $b$.

According to previous description, we can choose a BLF candidate as follows:

$$
V_{1}=\frac{1}{2}\left(\log \frac{k_{b 1}^{2}}{k_{b 1}^{2}-z_{1}^{2}}\right),
$$

where $\log (\cdot)$ denotes the natural logarithm. $k_{b 1}$ is the constraint on $z_{1}$ and satisfies the condition $\left|z_{1}\right|<k_{b 1}$. Thus it can be seen that $V_{1}$ is positive definite, which grows to infinity when its argument approaches to its finite limit $k_{b 1}$.

Lemma 3 (see [24]). For function $\sum_{k=1}^{p} \bar{f}_{k 1}\left(x_{1}\right) \theta_{f_{k 1}}^{\sigma(t)}$, a smooth function $h_{1}\left(x_{1}\right)$ and an unknown positive constant $l_{1}$ can be found:

$$
\left|\sum_{k=1}^{p} \bar{f}_{k 1}\left(x_{1}\right) \theta_{f_{k 1}}^{\sigma(t)}\right| \leq l_{1} h_{1}\left(x_{1}\right) .
$$

Lemma 4 (see [10]). For any positive constant $k_{b 1}$, any $z_{1}$ satisfying $\left|z_{1}\right|<k_{b 1}$, we have

$$
\log \frac{k_{b 1}^{2}}{k_{b 1}^{2}-z_{1}^{2}}<\frac{z_{1}}{k_{b 1}^{2}-z_{1}^{2}} .
$$

In order to make the problem more analysable and tractable, practical assumption is given for the adaptive state controller.

Assumption 5 (see [24]). We can find a smooth function $\bar{f}_{k 1}\left(x_{1}\right)$ such that

$$
f_{k 1}\left(x_{1}\right)=\bar{f}_{k 1}\left(x_{1}\right) z_{1}
$$

\section{Main Results}

In this section, an adaptive controller is designed based on backstepping method by utilizing a BLF for systems (3). 
Step 1. Define $z_{1}$ for $x_{1}$ as the tracking error which is $z_{1}=$ $x_{1}-y_{d}$. Consider the first component of the system (3) and we have the first-order partial derivative as

$$
\begin{aligned}
\dot{z}_{1} & =\dot{x}_{1}-\dot{y}_{d} \\
& =\sum_{k=1}^{p} f_{k 1}\left(x_{1}\right) \theta_{f_{k 1}}^{\sigma(t)}+\theta_{b}^{\sigma(t)} g_{1}\left(x_{1}\right) x_{2}-\dot{y}_{d},
\end{aligned}
$$

Choose the following BLF candidate:

$$
V_{1}=\frac{1}{2}\left(\log \frac{k_{b 1}^{2}}{k_{b 1}^{2}-z_{1}^{2}}+\widetilde{l}_{1}^{2}\right),
$$

where $\tilde{l}_{1}=l_{1}-\widehat{l}_{1}, \hat{l}_{1}$ denotes the estimate of $l_{1}$, and $\dot{\vec{l}}_{1}=-\dot{\vec{l}}_{1}$. We design a stabilizing function and an adaptive law as

$$
\begin{aligned}
& \alpha_{1}=\frac{1}{\bar{\theta}_{b} g_{1}}\left(-\widehat{l}_{1} h_{1} z_{1}-\lambda_{1} z_{1}+\dot{y}_{d}\right), \\
& \dot{\vec{l}}_{1}=\frac{z_{1}^{2} h_{1}}{k_{b 1}^{2}-z_{1}^{2}},
\end{aligned}
$$

where $\lambda_{1}$ is a positive gain. Besides, let $z_{2}=x_{2}-\alpha_{1}$. On the basis of (10) and Assumption 1, we know that the time derivate of $V_{1}$ satisfies

$$
\begin{aligned}
\dot{V}_{1} & =\frac{z_{1} \dot{z}_{1}}{k_{b 1}^{2}-z_{1}^{2}}+\tilde{l}_{1} \dot{\vec{l}}_{1}=\frac{z_{1}\left(\sum_{k=1}^{p} f_{k 1}\left(x_{1}\right) \theta_{f k 1}^{\sigma(t)}+\theta_{b}^{\sigma(t)} g_{1}\left(z_{2}+\alpha_{1}\right) \dot{y}_{d}\right)}{k_{b 1}^{2}-z_{1}^{2}}+\tilde{l}_{1} \dot{\vec{l}}_{1} \\
& \leq \frac{\left|z_{1}\right|^{2} l_{1} h_{1}+\theta_{b}^{\sigma(t)} g_{1} z_{1}\left(z_{2}+\left(1 / \bar{\theta}_{b} g_{1}\right)\left(-\widehat{l}_{1} h_{1} z_{1}-\lambda_{1} z_{1}+\dot{\bar{y}}_{d} z_{1}\right)\right)-\dot{\bar{y}}_{d}\left|z_{1}\right|^{2}}{k_{b 1}^{2}-z_{1}^{2}}+\tilde{l}_{1}\left(-\frac{\left|z_{1}\right|^{2} h_{1}}{k_{b 1}^{2}-z_{1}^{2}}\right) \leq-\frac{\lambda_{1} z_{1}^{2}}{k_{b 1}^{2}-z_{1}^{2}}
\end{aligned}
$$

where $\dot{\bar{y}}_{d}$ can be found that $\dot{y}_{d}=\dot{\bar{y}}_{d} z_{1}$.

Step 2. Consider the second component of system (3). Since $\dot{z}_{2}=\dot{x}_{2}-\dot{\alpha}_{1}$, we can find a $\dot{\bar{\alpha}}_{1}$ satisfying $\dot{\alpha}_{1}=\dot{\bar{\alpha}}_{1} z_{2}$ and choose the following BLF candidate:

$$
V_{2}=\sum_{i=1}^{2} \frac{1}{2}\left(\log \frac{k_{b i}^{2}}{k_{b i}^{2}-z_{i}^{2}}+\widetilde{l}_{i}^{2}\right) .
$$

The stabilizing function and the adaptive law at this step are designed as

$$
\begin{aligned}
& \alpha_{2}=\frac{1}{\bar{\theta}_{b} g_{2}}\left(-\hat{l}_{2} h_{2} z_{2}-\lambda_{2} z_{2}-\dot{\alpha}_{1}-\frac{k_{b 2}^{2}-z_{2}^{2}}{k_{b 1}^{2}-z_{1}^{2}} \bar{\theta}_{b} g_{1} z_{1}\right), \\
& \dot{\hat{l}}_{1}=\frac{z_{2}^{2} h_{2}}{k_{b 2}^{2}-z_{2}^{2}} .
\end{aligned}
$$

Using (13) and Assumption 1, we know that the time derivate of $V_{2}$ satisfies

$$
\begin{aligned}
\dot{V}_{2} & =\frac{z_{1} \dot{z}_{1}}{k_{b 1}^{2}-z_{1}^{2}}+\tilde{l}_{1} \dot{\vec{l}}_{1}+\frac{z_{2} \dot{z}_{2}}{k_{b 2}^{2}-z_{2}^{2}}+\tilde{l}_{2} \dot{\vec{l}}_{2} \leq-\frac{\lambda_{1} z_{1}^{2}}{k_{b 1}^{2}-z_{1}^{2}} \\
& +\frac{z_{1} z_{2} \theta_{b}^{\sigma(t)} g_{1}}{k_{b 1}^{2}-z_{1}^{2}}+\frac{z_{2} \dot{z}_{2}}{k_{b 2}^{2}-z_{2}^{2}}+\tilde{l}_{2} \dot{\tilde{l}}_{2} \leq-\frac{\lambda_{1} z_{1}^{2}}{k_{b 1}^{2}-z_{1}^{2}} \\
& +\frac{z_{1} z_{2} \theta_{b}^{\sigma(t)} g_{1}}{k_{b 1}^{2}-z_{1}^{2}} \\
& +\frac{z_{2}\left(\sum_{k=1}^{p} f_{k 2}\left(x_{2}\right) \theta_{f_{k 2}}^{\sigma(t)}+\theta_{b}^{\sigma(t)} g_{2}\left(x_{2}\right)\left(z_{3}+\alpha_{2}\right)-\dot{\alpha}_{1}\right)}{k_{b 2}^{2}-z_{2}^{2}} \\
& +\tilde{l}_{2} \dot{\vec{l}}_{2} \leq-\frac{\lambda_{2} z_{2}^{2}}{k_{b 2}^{2}-z_{2}^{2}}-\frac{\lambda_{1} z_{1}^{2}}{k_{b 1}^{2}-z_{1}^{2}} \leq 0 .
\end{aligned}
$$

For the general case, we employ the BLF when $n>2$ :

$$
V_{i}=\sum_{i=1}^{j} \frac{1}{2}\left(\log \frac{k_{b i}^{2}}{k_{b i}^{2}-z_{i}^{2}}+\widetilde{l}_{i}^{2}\right), \quad 2<j<n .
$$

By the same token, we design stabilizing functions and adaptive laws:

$$
\begin{aligned}
\alpha_{i} & =\frac{1}{\bar{\theta}_{b} g_{i}}\left(-\widehat{l}_{i} h_{i} z_{i}-\lambda_{i} z_{i}+\dot{\alpha}_{i-1}\right. \\
& \left.-\frac{k_{b i}^{2}-z_{i}^{2}}{k_{b(i-1)}^{2}-z_{i-1}^{2}} \bar{\theta}_{b} g_{i-1} z_{i-1}\right), \\
\dot{\vec{l}}_{1} & =\frac{z_{i}^{2} h_{i}}{k_{b i}^{2}-z_{i}^{2}} .
\end{aligned}
$$

Finally, we obtain the stabilizing function and the adaptive law of step $n$ :

$$
\begin{aligned}
& \alpha_{n} \\
& \quad=\frac{1}{\bar{\theta}_{b} g_{n}}\left(-\hat{l}_{n} h_{n} z_{n}-\lambda_{n} z_{n}+\dot{\alpha}_{n-1}-\frac{\bar{\theta}_{b} g_{n-1} z_{n-1}}{k_{b(n-1)}^{2}-z_{n-1}^{2}}\right), \\
& u=\alpha_{n}, \\
& \dot{\hat{l}}_{n}=z_{n}^{2} h_{n} .
\end{aligned}
$$

Theorem 6. Considering system (3), the adaptive state feedback controller (18) and the adaptive law (19) can guarantee $\lim _{t \rightarrow \infty}\left(x_{1}-y_{d}\right)=0$ and $\left|x_{i}\right| \leq b$ under arbitrary switching signal and $b$ is a positive constant. 


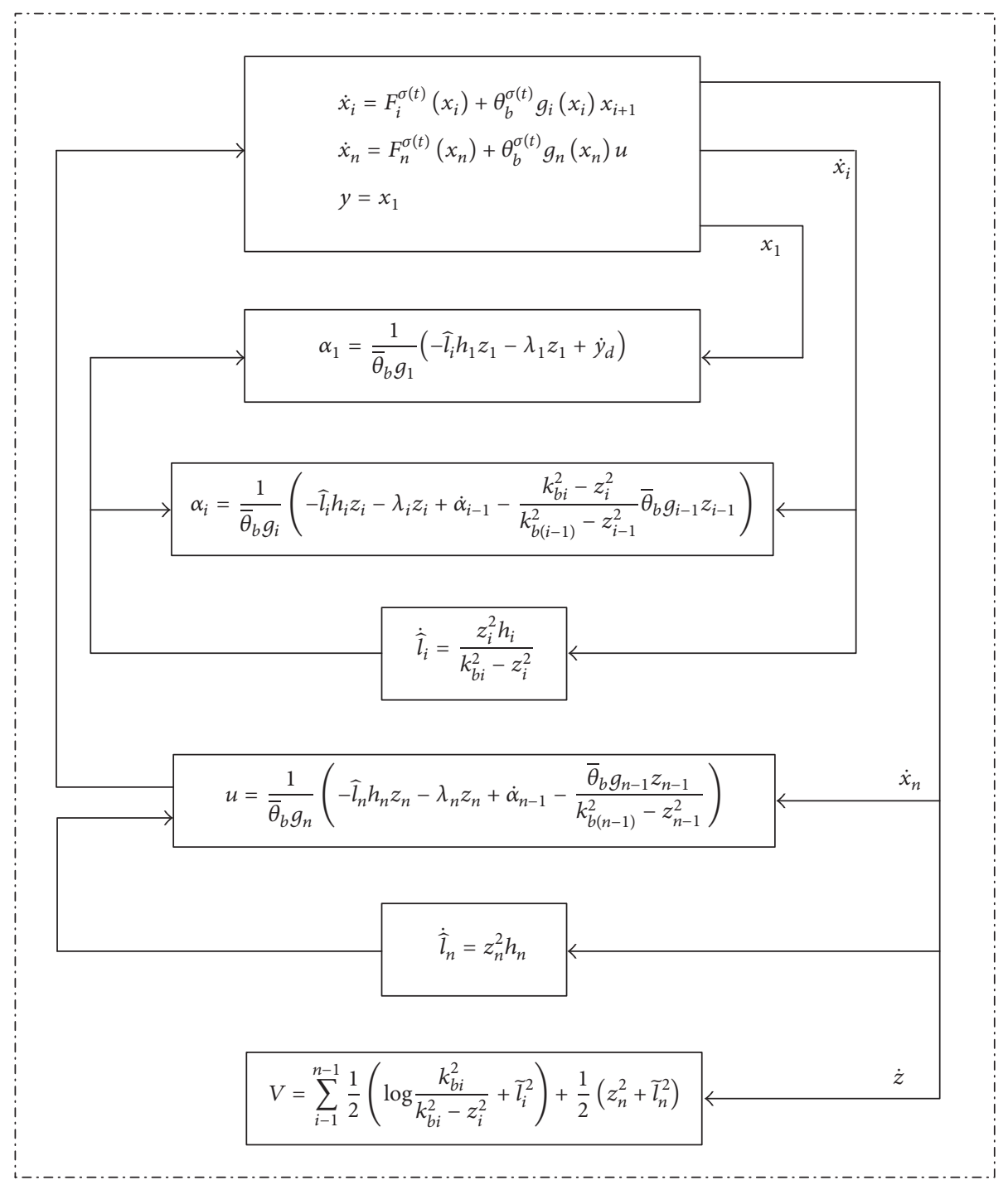

FIGURE 1: Block diagram of the adaptive control system.

Proof. Choose the BLF as follows:

$$
V=\sum_{i=1}^{n-1} \frac{1}{2}\left(\log \frac{k_{b i}^{2}}{k_{b i}^{2}-z_{i}^{2}}+\widetilde{l}_{i}^{2}\right)+\frac{1}{2}\left(z_{n}^{2}+\widetilde{l}_{n}^{2}\right) .
$$

According to the adaptive state feedback controller (18) and the adaptive law (19), we obtain the time derivative of the BLF:

$$
\begin{aligned}
\dot{V} & \leq-\sum_{i=1}^{n-1} \frac{\lambda_{i} z_{i}^{2}}{k_{b i}^{2}-z_{i}^{2}}+\frac{z_{n-1} z_{n} \theta_{b}^{\sigma(t)} g_{n-1}}{k_{b(n-1)}^{2}-z_{n-1}^{2}}+z_{n} \dot{z}_{n}+\tilde{l}_{n} \dot{\vec{l}}_{n} \\
& \leq-\sum_{i=1}^{n} \frac{\lambda_{i} z_{i}^{2}}{k_{b i}^{2}-z_{i}^{2}} \leq 0 .
\end{aligned}
$$

It can be seen that all the signals are bounded. By Barbalat's Lemma, $z_{i}$ will converge to zero. According to the nature of BLF, we can guarantee $\lim _{t \rightarrow \infty}\left(x_{1}-y_{d}\right)=0$ and $\left|x_{i}\right| \leq b$, among them $b$ being a positive constant. The output of the system tracks the desired trajectory $y_{d}$ asymptotically and certain states are constrained.

The design procedure of the proposed control scheme could be viewed from the block diagram in Figure 1.

\section{Simulations}

In this section, we give examples and simulations to demonstrate the proposed result.

Example 1. Consider the following switched nonlinear system:

$$
\begin{gathered}
\dot{x}_{1}=\left[\begin{array}{lll}
f_{11} & f_{12} & f_{13}
\end{array}\right] \theta_{1}^{\sigma}+\theta_{b}^{\sigma} g_{1}\left(x_{1}\right) x_{2} \\
\dot{x}_{2}=\left[\begin{array}{lll}
f_{21} & f_{22} & f_{23}
\end{array}\right] \theta_{2}^{\sigma}+\theta_{b}^{\sigma} g_{2}\left(x_{2}\right) u, \\
\sigma(t):[0, \infty) \longrightarrow\{1,2\},
\end{gathered}
$$




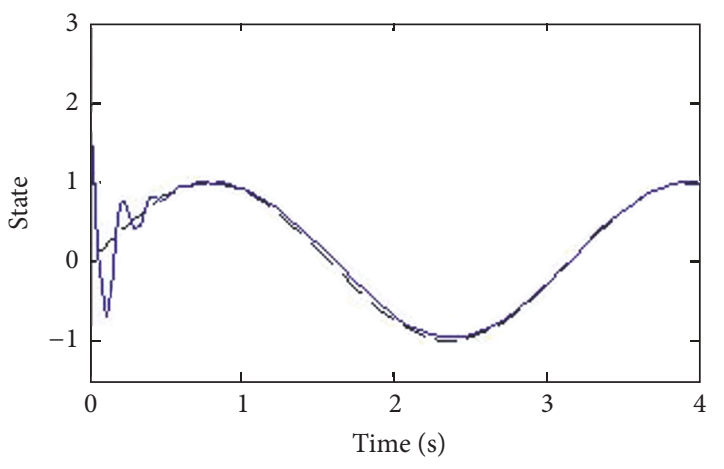

FIGURE 2: State trajectory $x_{1}$ tracking converges the desired trajectory $y_{d}=\sin x$.

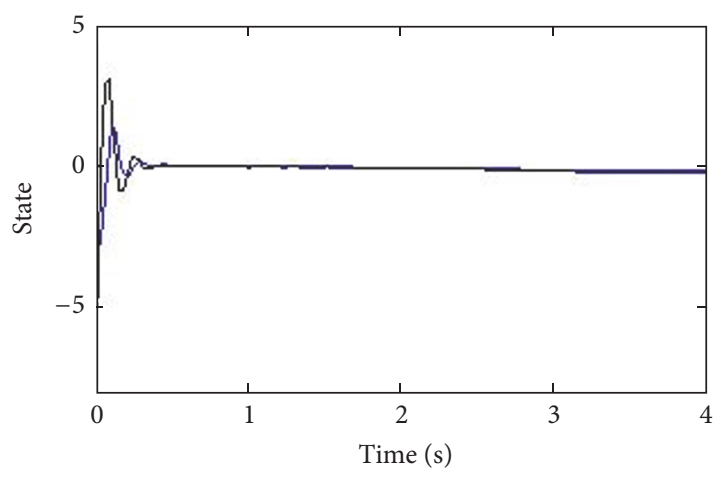

FIGURE 3: State trajectory $x_{2}$ tracking converges the desired trajectory $\alpha_{1}$.

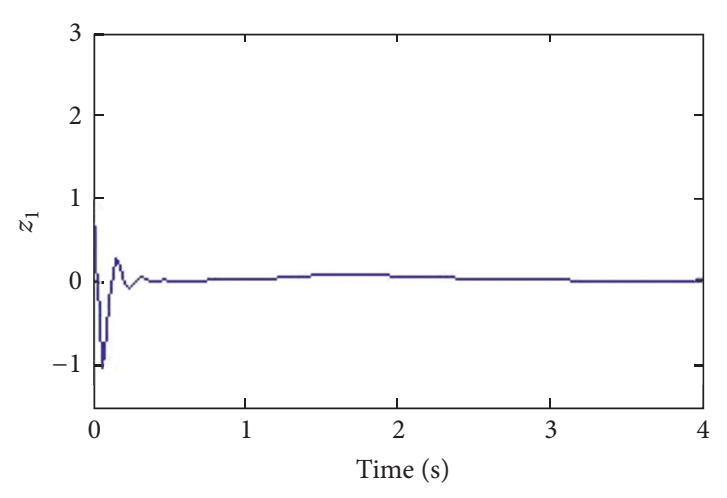

FIGURE 4: Error trajectory $z_{1}$ converges to zero.

where $\left[\begin{array}{lll}f_{11} & f_{12} & f_{13}\end{array}\right]=\left[\begin{array}{lll}x_{1}^{2} & x_{1} & 1\end{array}\right],\left[\begin{array}{lll}f_{21} & f_{22} & f_{23}\end{array}\right]=$ $\left[\begin{array}{lll}x_{2}^{2} & x_{2} & \cos \left(x_{2}\right)\end{array}\right], g_{1}\left(x_{1}\right)=2 x_{1}^{2}+2, g_{2}\left(x_{2}\right)=x_{2}+3$. Parameters $\theta_{1}^{\sigma}, \theta_{2}^{\sigma}$, and $\theta_{b}^{\sigma}$ are unknown. According to previous discussions, we can find smooth functions and satisfy the conditions of Theorem 6 that

$$
\begin{aligned}
& h_{1}\left(x_{1}\right)=2 x_{1}^{2}+5, \\
& h_{2}\left(x_{2}\right)=x_{2}^{2}+2 .
\end{aligned}
$$

The desired trajectory is $y_{d}=\sin x$, and the tracking error constraint is $\left|z_{1}\right|<2,\left|z_{2}\right|<10$.

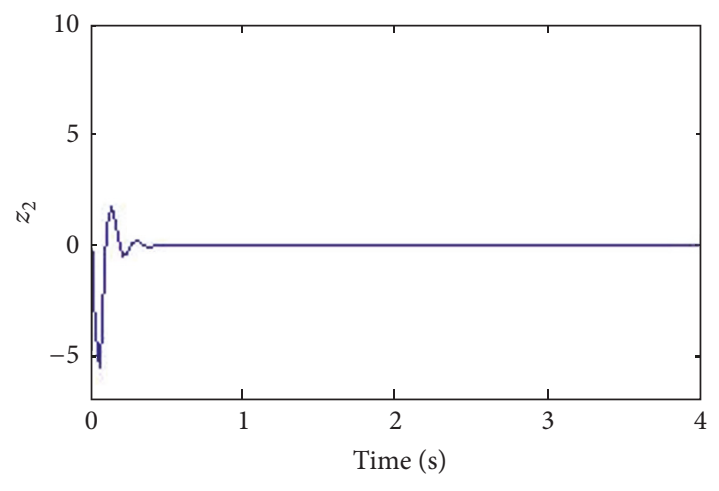

FIGURE 5: Error trajectory $z_{2}$ converges to zero.

Figures 2 and 3 present the simulation results of the outputs of the system tracking the desired trajectory $y_{d}$ asymptotically, and the tracking errors constraints are never violated. And it is evident from Figures 4 and 5 that tracking error trajectories converge to zero.

After that, we design a controller using QLF. Choose $V_{1}=$ $(1 / 2)\left(z_{1}^{2}+\widetilde{l}_{1}^{2}\right)$, and we can show that $\phi_{1}$ is a common virtual control for the first component of the system (22). Then, define $z_{2}$ for $x_{2}$ as the tracking error which $z_{2}=x_{2}-\phi_{1}$, $V_{2}=\sum_{i=1}^{2}(1 / 2)\left(z_{2}^{2}+\widetilde{l}_{2}^{2}\right)$ is a common Lyapunov for the system (22). We will follow the same design procedure for the design of the controller based on the QLF method and compare the simulation results between BLF and QLF.

As it can be clearly seen from Figures 6 and 7, we know that the asymptotic tracking performance is achieved and $x_{1}$ tracks the desired trajectory $y_{d}$ asymptotically based on BLF method. However, when the QLF is utilized with the same parameters and design processes, the output is more volatile than the BLF one, and the convergence rate is slower than the BLF method.

Example 2. Considering the above system, the control objective is that the output of system $x_{1}$ tracks the desired trajectory $y_{d}=0.3$. Two different tracking error constraints are given that $\left|z_{1}\right|<0.2,\left|z_{1}\right|<0.15$.

From Figure 8, we know that asymptotic tracking performance is achieved when the BLF is used with the initial value $x(0)=(0.25,-1)^{T}$. However, when the QLF is used with the same initial value, the state constraint is violated. The simulation results in Figures 9 and 10 show that we can know that tracking errors $z_{1}$ satisfy constraints using the BLF. The errors $z_{1}$ do not transgress boundary that $\left|z_{1}\right|<0.2$ and $\left|z_{1}\right|<0.15$, and the objective is achieved.

\section{Conclusions}

In this paper, we have studied the constraint adaptive tracking control problem of switched nonlinear systems with uncertain parameters using the BLF and backstepping method. Asymptotic output tracking and states constraint have been ensured, and we guarantee that the state constraint is violated, which has been verified by the simulations. Next we will focus on studying constraint adaptive output feedback control and 


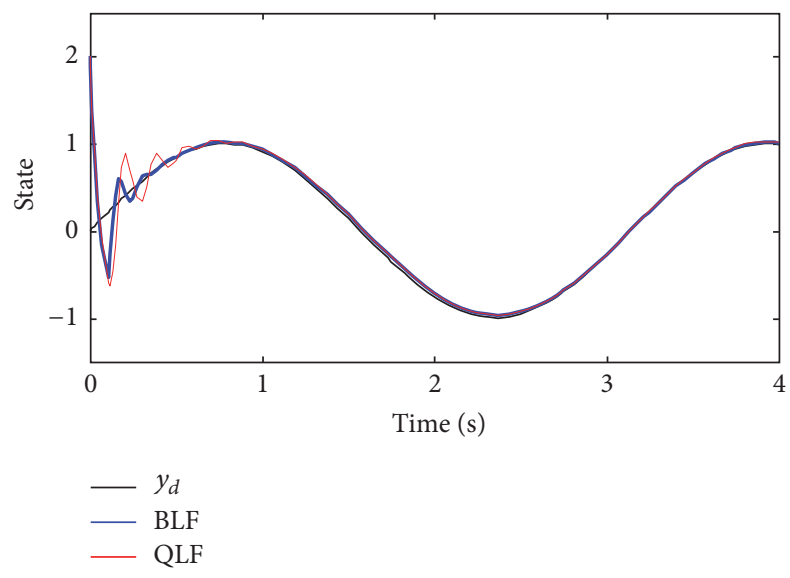

FIGURE 6: State trajectories $x_{1}$ tracking converge the desired trajectory (BLF-QLF).

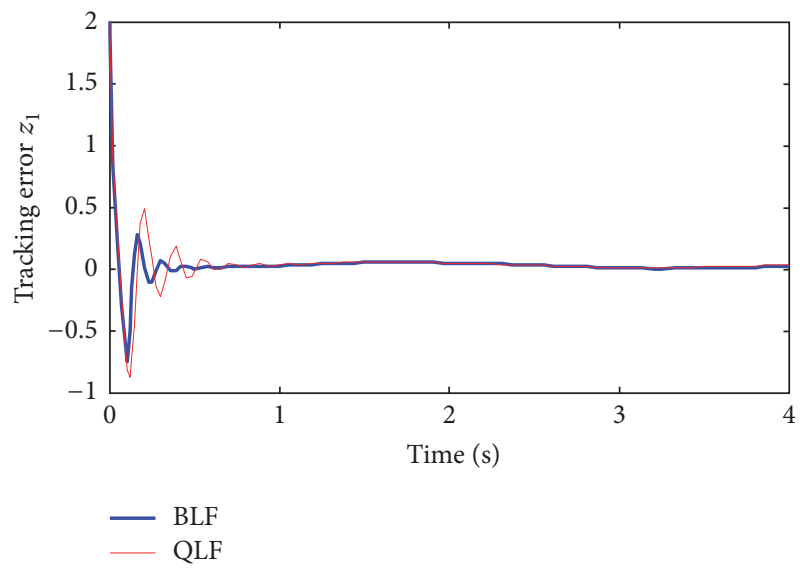

FIgURE 7: Error trajectories $z_{1}$ converge to zero (BLF-QLF).

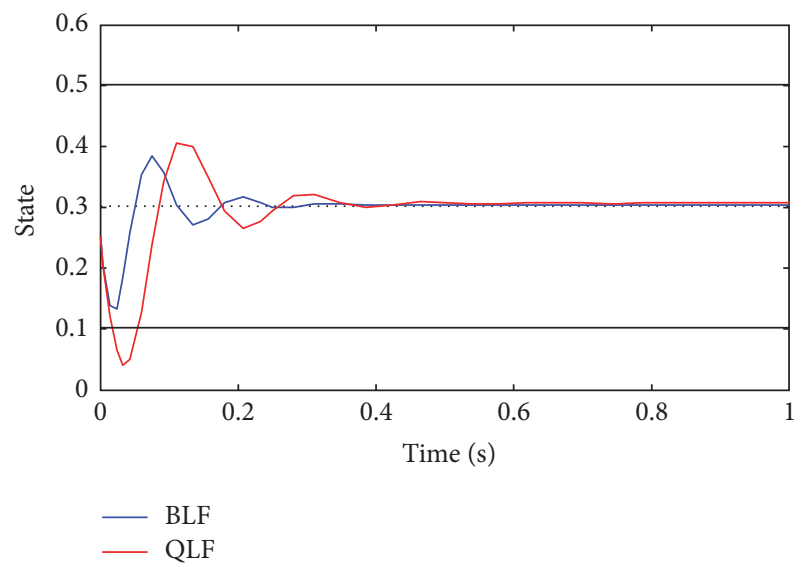

Figure 8: State trajectories $x_{1}$ tracking converges the desired trajectory $\left(\left|z_{1}\right|<0.2\right)$.

designing an adaptive controller and stabilization under arbitrary switching signals can be achieved by output feedback.

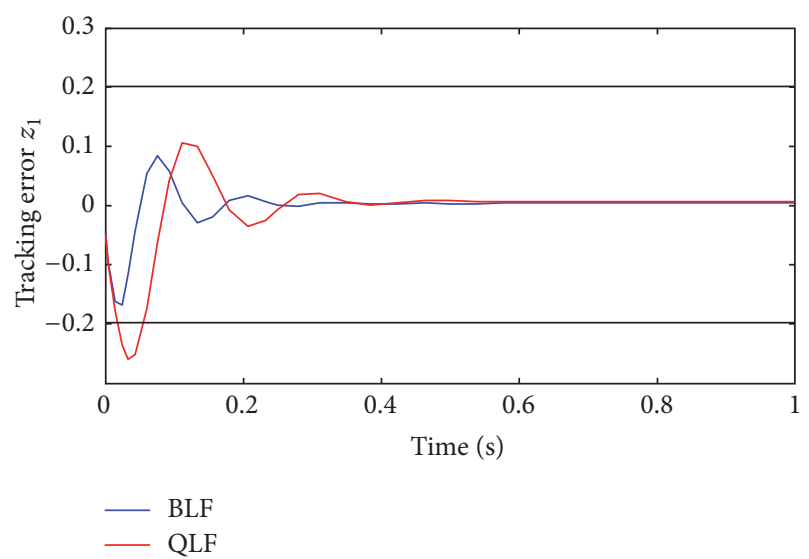

FIgURE 9: Tracking error trajectories $z_{1}$ satisfies error constraint $\left|z_{1}\right|<0.2$.

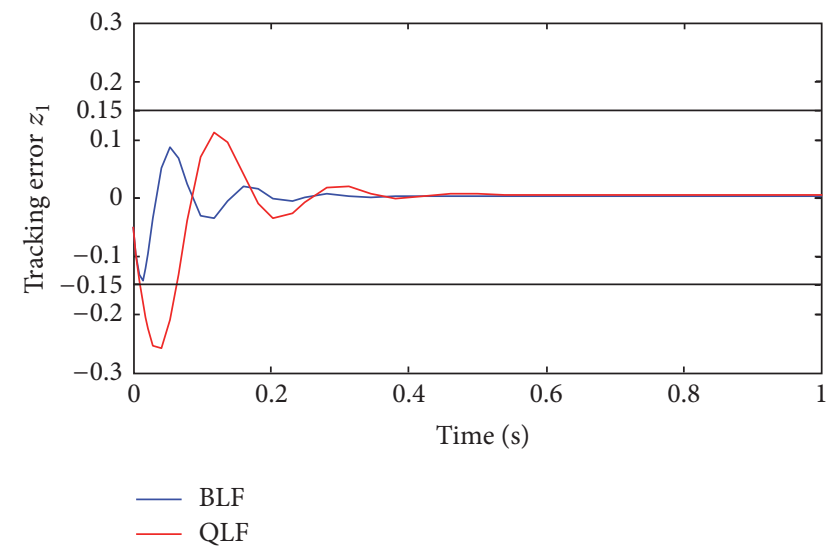

FIGURE 10: Tracking error trajectories $z_{1}$ satisfies error constraint $\left|z_{1}\right|<0.15$.

\section{Conflicts of Interest}

The authors declare that there are no conflicts of interest regarding the publication of this paper.

\section{References}

[1] J. C. Geromel and P. Colaneri, "Stability and stabilization of continuous-time switched linear systems," SIAM Journal on Control and Optimization, vol. 45, no. 5, pp. 1915-1930, 2006.

[2] Z. Ji, L. Wang, and G. Xie, "Quadratic stabilization of switched systems," International Journal of Systems Science. Principles and Applications of Systems and Integration, vol. 36, no. 7, pp. 395404, 2005.

[3] Q.-K. Li, J. Zhao, and G. M. Dimirovski, "Robust tracking control for switched linear systems with time-varying delays," IET Control Theory and Applications, vol. 2, no. 6, pp. 449-457, 2008.

[4] G. Chesi, "On the minimum stable commutation time for switching nonlinear systems," Institute of Electrical and Electronics Engineers. Transactions on Automatic Control, vol. 54, no. 6, pp. 1284-1289, 2009. 
[5] L.-L. Li, J. Zhao, and G. M. Dimirovski, "Robust Hoocontrol for a class of switched non-linear systems with neutral uncertainties," Transactions of the Institute of Measurement and Control, vol. 32, no. 6, pp. 635-659, 2010.

[6] X. Zhao, S. Yin, H. Li, and B. Niu, "Switching stabilization for a class of slowly switched systems," Institute of Electrical and Electronics Engineers. Transactions on Automatic Control, vol. 60, no. 1, pp. 221-226, 2015.

[7] R. Ma and J. Zhao, "Backstepping design for global stabilization of switched nonlinear systems in lower triangular form under arbitrary switchings," Automatica. A Journal of IFAC, the International Federation of Automatic Control, vol. 46, no. 11, pp. 1819-1823, 2010.

[8] F. Blanchini, "Set invariance in control," Automatica. A Journal of IFAC, the International Federation of Automatic Control, vol. 35, no. 11, pp. 1747-1767, 1999.

[9] K. Kogiso and K. Hirata, "Reference governor for constrained systems with time-varying references," Robotics and Autonomous Systems, vol. 57, no. 3, pp. 289-295, 2009.

[10] B. Ren, S. S. Ge, K. P. Tee, and T. H. Lee, "Adaptive neural control for output feedback nonlinear systems using a barrier lyapunov function," IEEE Transactions on Neural Networks, vol. 21, no. 8, pp. 1339-1345, 2010.

[11] K. P. Tee and S. S. Ge, "Control of nonlinear systems with full state constraint using a barrier lyapunov function," in Proceedings of the 48th IEEE Conference on Decision and Control Held Jointly with 28th Chinese Control Conference (CDC/CCC '09), pp. 8618-8623, Shanghai, China, December 2009.

[12] K. P. Tee, S. S. Ge, and E. H. Tay, "Barrier Lyapunov functions for the control of output-constrained nonlinear systems," Automatica. A Journal of IFAC, the International Federation of Automatic Control, vol. 45, no. 4, pp. 918-927, 2009.

[13] K. P. Tee and S. S. Ge, "Control of nonlinear systems with partial state constraints using a barrier lyapunov function," International Journal of Control, vol. 84, no. 12, pp. 2008-2023, 2011.

[14] B. Niu and J. Zhao, "Tracking control for output-constrained nonlinear switched systems with a barrier Lyapunov function," International Journal of Systems Science. Principles and Applications of Systems and Integration, vol. 44, no. 5, pp. 978-985, 2013.

[15] H. Q. Wang, W. J. Sun, and P. X. Liu, "Adaptive intelligent control of nonaffine nonlinear time-delay systems with dynamic Uncertainties," IEEE Transactions on Systems Man Cybernetics Systems, pp. 1-12, 2016.

[16] H. Wang, P. X. Liu, and P. Shi, “Observer-Based Fuzzy Adaptive Output-Feedback Control of Stochastic Nonlinear Multiple Time-Delay Systems," IEEE Transactions on Cybernetics, vol. 99, pp. 1-11, 2017.

[17] X. Zhao, H. Yang, H. R. Karimi, and Y. Zhu, "Adaptive neural control of MIMO nonstrict-feedback nonlinear systems with time delay," IEEE Transactions on Cybernetics, vol. 46, no. 6, pp. 1337-1349, 2015.

[18] T. T. Han, S. S. Ge, and T. H. Lee, "Adaptive neural control for a class of switched nonlinear systems," Systems Control Letters, vol. 58, pp. 109-118, 2009.

[19] W. Lin and C. Qian, "Adaptive control of nonlinearly parameterized systems: a nonsmooth feedback framework," Institute of Electrical and Electronics Engineers. Transactions on Automatic Control, vol. 47, no. 5, pp. 757-774, 2002.

[20] Q. Sang and G. Tao, "Adaptive control of piecewise linear systems: the state tracking case," Institute of Electrical and Electronics Engineers. Transactions on Automatic Control, vol. 57, no. 2, pp. 522-528, 2012.
[21] M.-L. Chiang and L.-C. Fu, "Variable structure based switching adaptive control for a class of unknown switched linear systems," in Proceedings of the 2009 American Control Conference, ACC 2009, pp. 3019-3024, usa, June 2009.

[22] X. Wang, J. Zhao, and Y. Tang, "State tracking model reference adaptive control for switched nonlinear systems with linear uncertain parameters," Journal of Control Theory and Applications, vol. 10, no. 3, pp. 123-127, 2012.

[23] X. Zhao, P. Shi, X. Zheng, and L. Zhang, "Adaptive tracking control for switched stochastic nonlinear systems with unknown actuator dead-zone," Automatica. A Journal of IFAC, the International Federation of Automatic Control, vol. 60, pp. 193-200, 2015.

[24] M.-L. Chiang and L.-C. Fu, "Adaptive stabilization of a class of uncertain switched nonlinear systems with backstepping control," Automatica. A Journal of IFAC, the International Federation of Automatic Control, vol. 50, no. 8, pp. 2128-2135, 2014. 


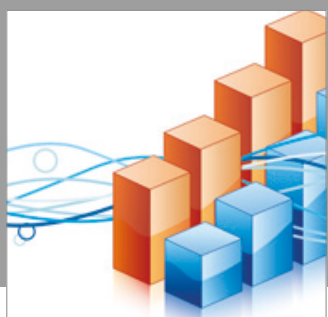

Advances in

Operations Research

vatersals

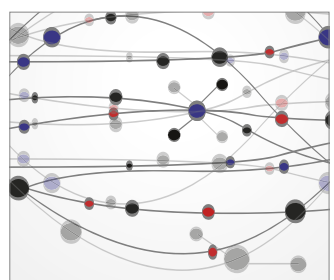

\section{The Scientific} World Journal
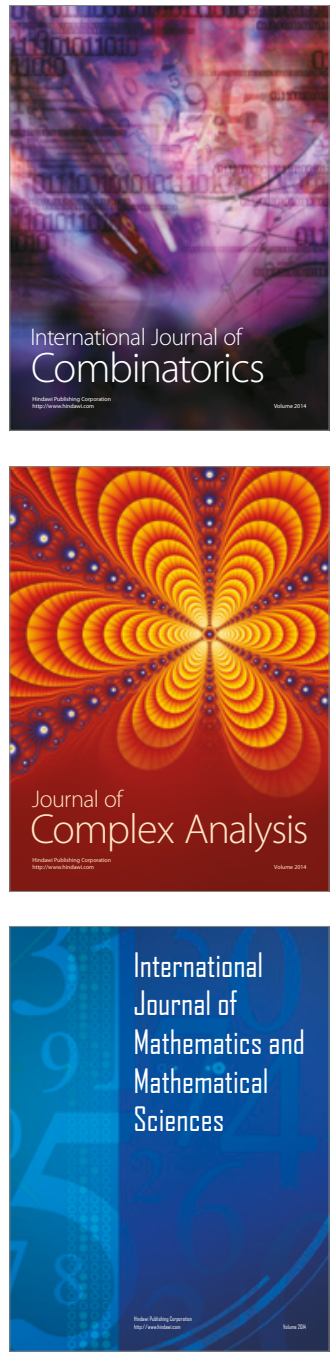
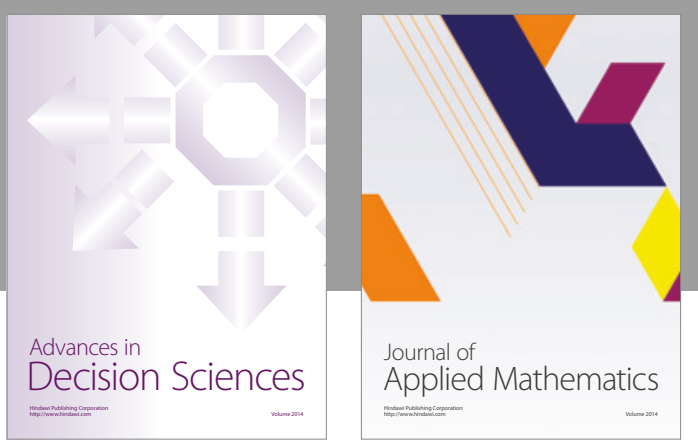

Algebra

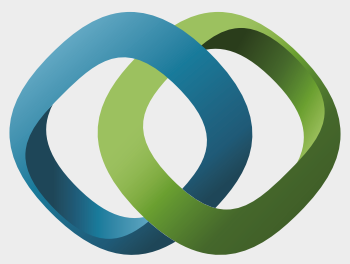

\section{Hindawi}

Submit your manuscripts at

https://www.hindawi.com
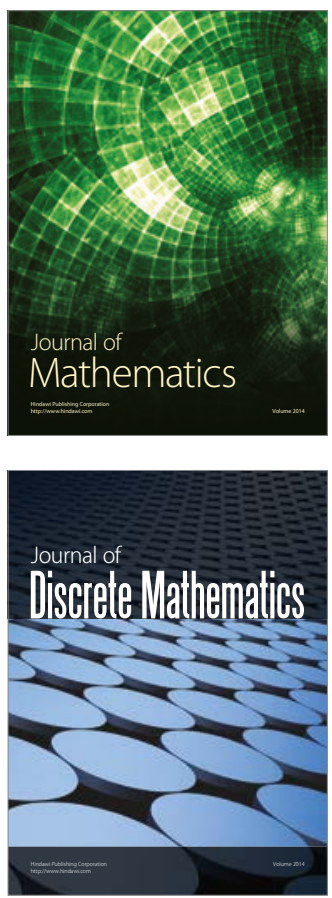

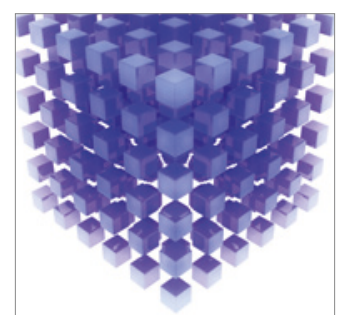

Mathematical Problems in Engineering
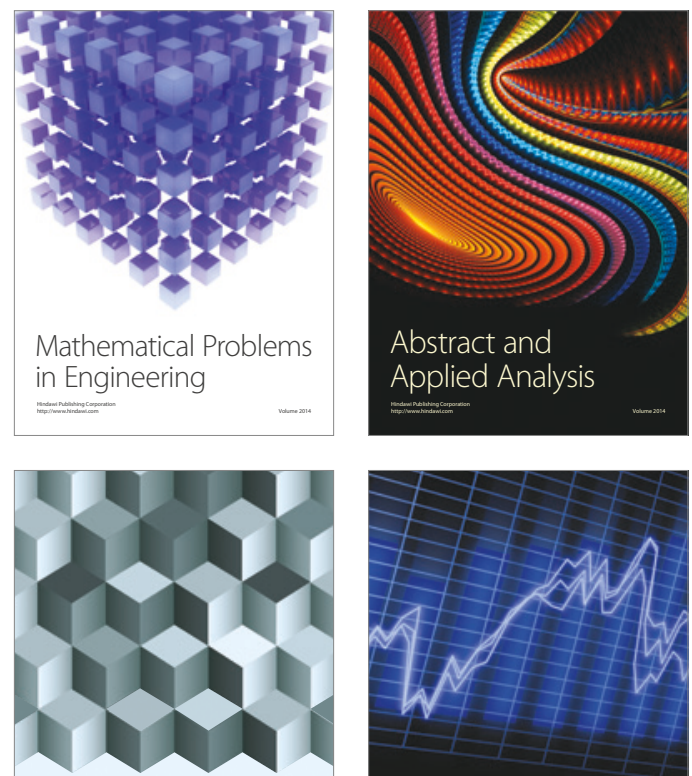

Journal of

Function Spaces

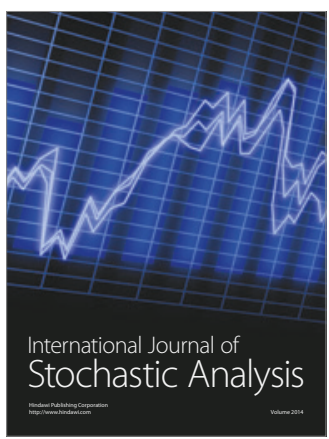

Probability and Statistics
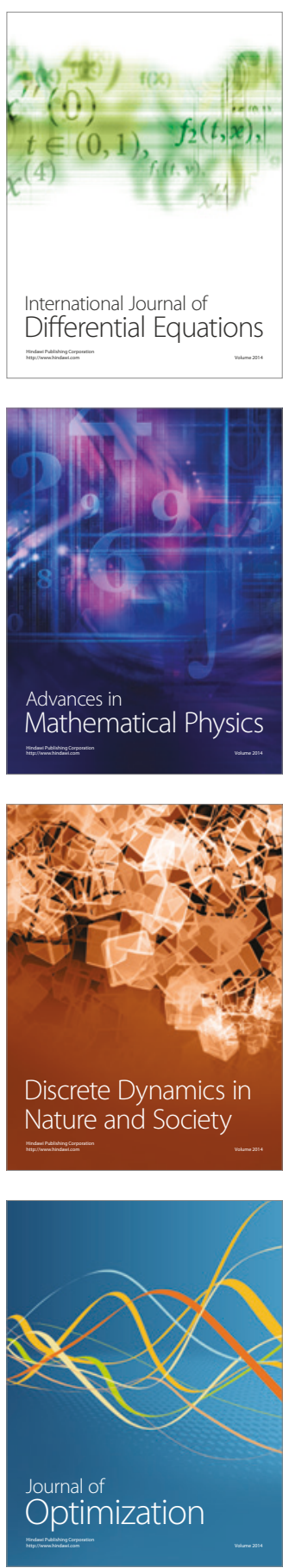